\title{
Entanglement localization by a single defect in a spin chain
}

\author{
Tony J. G. Apollaro ${ }^{1,2}$ and Francesco Plastina ${ }^{1,2}$ \\ 1 Dipartimento di Fisica, Università della Calabria, 87036 Arcavacata di Rende (CS) Italy \\ ${ }^{2}$ INFN - Gruppo collegato di Cosenza, 87036 Arcavacata di Rende (CS) Italy
}

(Dated: May 14, 2019)

\begin{abstract}
We discuss the effect of a single diagonal defect on both the static and dynamical properties of entanglement in a spin chain. We show that entanglement localizes at the defect and discuss its localization length, arguing that this can be used as a mean to store entanglement. We also show that the impurity site can behave as an entanglement mirror and characterize the bouncing process in terms of reflection and transmission coefficients.
\end{abstract}

PACS numbers: 03.67.Hk, 03.67.Mn, 05.50+q

Entanglement generation and distribution is a problem of central importance in performing quantuminformation tasks, like teleportation and quantum cryptography. As entanglement between parties is created by means of a direct interaction, methods are required to transfer either the entangled particles or their state. Indeed, the problem of designing quantum networks enabling efficient high-fidelity transfer of quantum states has been addressed by a number of authors, [1, 2, 3, 4, 5, 6], especially focusing on the requirement of minimal control. This means that state transfer should be possible without performing many control operations such as switching on and off the interactions, measuring, or applying encoding and decoding procedures.

In this respect, spin systems provide ideal models to study entanglement properties as they are naturally employed as qubit registers and exploited as quantum channels (or coherent data bus). Spin chains with fixed interactions have been considered [1], and solid state implementations have been already proposed 2]. It has also been shown that perfect transfer can be achieved by performing local measurements [3], by using several spin chains in parallel [4], by employing local memories at the receiver side 5], or by means of a spatial modulation of the spin coupling strengths $[\underline{6}]$. For this latter case, the effect of imperfections (static errors) in the engineering of the spin system has been analyzed in Ref. 7], where a scaling of the transmission fidelity has been obtained in terms of the degree of imperfection. This is an example in which the disorder has been taken into account. In fact, considerable attention has been devoted to disordered spin systems. A threshold in the coupling has been found for the onset of entanglement between a bulk impurity and its neighborhood [8]. Also it has been shown that a faster (super-ballistic) distribution of entanglement between a central node and a series of distributed sites occurs if the sender resides in a disordered region $[9]$. Furthermore, perturbative, numerical, and Bethe-ansatzbased investigations of entanglement between two defects has been performed for a disordered anti-ferromagnetic spin-1/2 chain with anisotropic exchange coupling, 10]. A possibility of tuning the ground state entanglement by a single off-diagonal impurity in the anisotropic $X Y$ model has also been considered 11].

Diagonal disorder has also been considered in the quantum information context, but only the wave function localization issue has been addressed in terms of the inverse participation ratio 12, while entanglement localization has been completely overlooked, apart from some considerations reported in Ref. [13].

It is well known that disorder can lead to a spatial localization of the electronic wave function [14] and that, in particular, this occurs whenever impurities exist in a tight binding model. In fact, a single impurity suffices [15] and, in this paper, we discuss how this translates into an entanglement localization.

We consider the simplest example of a system displaying localization; namely, a spin chain with a single diagonal defect, that is, a chain subject to a local static magnetic field which is equal at every spin location except for a single site, where a field defect is present. We analyze both the static and dynamical properties of such a system, and, in particular, we discuss the case in which the ground state of the chain is a localized state, showing a very peculiar spatial distribution of quantum correlations. We also discuss how this localized state affects the possibility of sending entanglement through such a 'defected' channel. In particular, we show that a mirror-like effect occurs at the defect during entanglement propagation. This achieves a (partial) control of the entanglement dynamics, as, starting from a homogeneous system, the modification of the single-qubit level spacing permits the generation of an "artificial defect".

The model we study is a 1-D isotropic XY spin- $\frac{1}{2}$ closed chain, placed in an external magnetic field which is homogeneous everywhere but for a single (defect) site $l$

$$
H=h \sum_{i=-\frac{N}{2}}^{\frac{N}{2}} \sigma_{z}^{i}-J \sum_{i=-\frac{N}{2}}^{\frac{N}{2}}\left(\sigma_{x}^{i} \sigma_{x}^{i+1}+\sigma_{y}^{i} \sigma_{y}^{i+1}\right)+\epsilon \sigma_{z}^{l}
$$

where $h$ is the magnetic field, acting on every site but for site $l$, where the field is $h+\epsilon$. Also, $J$ is a ferromagnetic coupling constant, while $\sigma_{\alpha}$ are the Pauli matrices. We will consider the impurity term as a perturbation and 
take as a reference the results obtained for the homogeneous XY Hamiltonian [16, 17]. Since $\left[H, \sum_{i} \sigma_{z}^{i}\right]=0$, the total Hilbert space can be divided into invariant subspaces labelled by the number of spins which point upwards. We will concentrate on the 0 and 1-excitation subspaces and denote by $|n\rangle$ the state with the upward spin at site $n$.

Thorough, we consider the case $2 h>J$. This implies that the unperturbed problem $(\epsilon=0)$ has the factorized ground state $|0\rangle^{\otimes N+1}$, whose energy we re-scale to zero. In the Hilbert space sector with one reversed spin, the unperturbed energy basis is

$$
|k\rangle=\frac{1}{\sqrt{N+1}} \sum_{n} \exp \left[\frac{2 \pi i k n}{N+1}\right]|n\rangle
$$

with energy $E_{k}=2 h-J \cos \left(\frac{2 \pi k}{N+1}\right)$, and where $k=$ $-\frac{N}{2},-\frac{N}{2}+1, \ldots, \frac{N}{2}$.

In this sector, the Hamiltonian (1) is equivalent to a tight binding problem with one diagonal impurity. This can be solved with the help of the Green's function technique [15].

The unperturbed Green operator is

$$
G_{0}(z)=\frac{1}{z-H_{0}}=\sum_{k} \frac{|k\rangle\langle k|}{z-E_{k}} .
$$

In the thermodynamic limit $(N \rightarrow \infty)$, the index $k$ becomes continuous and the states $|k\rangle$ give rise to a continuous energy band, with $E_{k} \in[2 h-J, 2 h+J]=I_{b}$. In this continuum limit, the matrix elements of $G_{0}$ between localized states can be written

$$
\begin{aligned}
& G_{0}(r, s ; z)=\frac{\left(-x+\sqrt{x^{2}-1}\right)^{|r-s|}}{J \sqrt{x^{2}-1}}, z \neq I_{b} \\
& G_{0}^{ \pm}(r, s ; z)=\frac{\left(-x \pm i \sqrt{1-x^{2}}\right)^{|r-s|}}{ \pm i J \sqrt{1-x^{2}}}, z \in I_{b}
\end{aligned}
$$

where $x=\frac{z-2 h}{J}$.

The Green operator for the full hamiltonian can be expressed in terms of the unperturbed one, and, due to the simple form of the interaction hamiltonian, the Dyson series can be re-summed giving an exact expression [15]

$$
G(z)=G_{0}(z)+G_{0}(z)|l\rangle \frac{2 \epsilon}{1-2 \epsilon G_{0}(l, l ; z)}\langle l| G_{0}(z) .
$$

The knowledge of the Green function (6) allows us to obtain the spectrum of the hamiltonian (10). It turns out that the energy band of the continuous spectrum is unaffected, as for $E \in[2 h-J, 2 h+J]$ the Green function has a cut in the complex plane. The associated eigenstates are given by $|\Psi(E)\rangle=\sum_{n} a_{n}(E)|n\rangle$, with

$$
a_{n}(E)=\frac{1}{\sqrt{N+1}}\left(e^{i \theta n}+\frac{\alpha e^{i|\theta||n-l|}}{i|\sin \theta|-\alpha} e^{i \theta l}\right),
$$

where $\alpha=\frac{2 \epsilon}{J}$ and $\cos \theta=(2 h-E) / J$.

Besides a distortion of the states within the band, the perturbation produces the appearance of a localized eigenstate, whose energy is given by the pole of the Green function (6), determined by the equation $2 \epsilon G_{0}\left(l, l ; E_{l o c}\right)=1$. This state lies above or below the band, depending on wether $\alpha$ is greater than zero or not, and its energy is $E_{l o c}=2 h \mp J \sqrt{1+\alpha^{2}}$. Explicitly, the state is given by $\left|\Psi_{l o c}\right\rangle=\sum_{n} b_{n}|n\rangle$, with

$$
b_{n}= \begin{cases}-\frac{\sqrt{|\alpha|}}{\left(1+\alpha^{2}\right)^{\frac{1}{4}}} \exp [-\xi(\alpha)|n-l|] & \alpha<0 \\ (-1)^{|n-l|} \frac{\sqrt{|\alpha|}}{\left(1+\alpha^{2}\right)^{\frac{1}{4}}} \exp [-\xi(\alpha)|n-l|] & \alpha>0\end{cases}
$$

where $\xi(\alpha)=-\ln \left(\sqrt{1+\alpha^{2}}-|\alpha|\right) . \quad \xi^{-1}$ is the spatial extension (or localization length) of $\left|\Psi_{l o c}\right\rangle$ around the impurity site $l$ ( $\xi$ is obtained in Ref. 18 for the $X X Z$ model). For $\frac{2 h}{J \sqrt{1+\alpha^{2}}}<1$ with $\alpha<0$ (that is, for negative and large enough $\alpha), E_{l o c}$ is lower than zero and $\left|\Psi_{l o c}\right\rangle$ becomes the ground state of the system.

We analyze the entanglement content of this localized state. In particular, we concentrate on the pairwise quantum correlations shared by the qubits at sites $i$ and $j$ as measured by the concurrence [19]. It is easy to show that the concurrence between these sites is given by the residue of the associated matrix element of the Green operator (to simplify the notation, we set $l=0$ and measure distances along the chain from the impurity site)

$$
C_{i j}=2 \operatorname{Res}\left[G\left(i, j ; E_{l o c}\right)\right]=\frac{2|\alpha|}{\sqrt{1+\alpha^{2}}} e^{-\xi(|i|+|j|)} .
$$

As a result of the presence of the magnetic field defect, pairwise entanglement is only present for spins residing within a distance $1 / \xi$ from the impurity and it decays exponentially with the separation. In this sense, we state that entanglement is localized in the neighborhood of the defect. As the localization length becomes less than the site spacing for $\alpha \leq\left(1-e^{2}\right) / 2 e \simeq-1.175$, entanglement is highly localized even for small values of the defect field.

We now analyze the transmission of a quantum state along the chain. In particular, we are interested in characterizing entanglement distribution; namely, the possibility of using the chain to send one partner of a maximally entangled pair. A related problem is the transmission of a single qubit state along the chain. To send one qubit, we imagine that at site $s$, the sender prepares a generic single qubit state $\left|\psi_{s}\right\rangle$ which is aimed to be retrieved at site $r$ (where, in general, the mixed state $\rho_{r}(t)$ is extracted at time $\left.t\right)$. A measure of the quality of the chain as a transmission channel is given by fidelity, $F(t)=\left\langle\psi_{s}\left|\rho_{r}(t)\right| \psi_{s}\right\rangle$, averaged over all possible messages $\left|\psi_{s}\right\rangle$. On the other hand, to send entanglement, the spin $s$ is initially prepared in a singlet state with an external (un-coupled) qubit. The entanglement transmission can be characterized by the (final) concurrence between the 


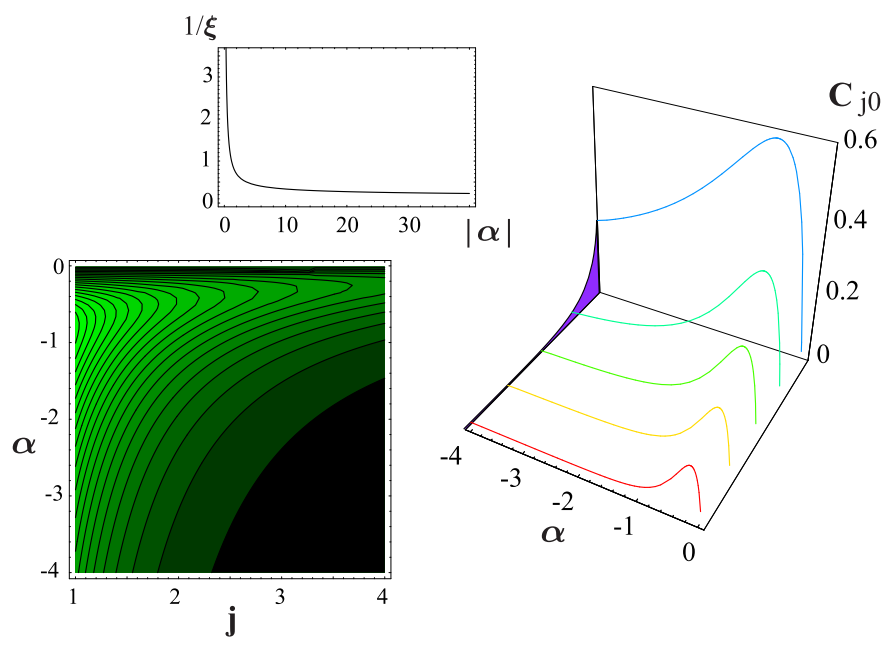

FIG. 1: (up-left) Localization length as a function of $|\alpha|$. (down-left and right) Ground state concurrence between the impurity and the spin at site $j$. Notice the exponential decrease as a function of the distance.

external qubit and the one residing at the receiving site $r$, denoted by $C_{r}(t)$.

In fact, the two quantities $F$ and $C$ are related [1], as both can be expressed in terms of the transmission amplitude to send an excitation from $s$ to $r, f_{r s}(t)=$ $\left\langle r\left|e^{-i H t}\right| s\right\rangle$. Explicitly, the averaged fidelity is $\langle F\rangle(t)=$ $\left(\left|f_{r s}(t)+1\right|^{2}+2\right) / 6$, whereas the concurrence is given by $C_{r}(t)=\left|f_{r s}\right|$. In our case, the transition amplitude is given by a complicated combination of matrix elements of the Green functions,

$$
\begin{gathered}
f_{r s}(t)=\int_{-\pi}^{\pi} \frac{d \theta}{2 \pi}\left\{e^{i \theta(r-s)}+e^{i \theta(l-s)} g_{r, l}^{(+)}+e^{i \theta(r-l)} g_{l, s}^{(-)}\right. \\
\left.+g_{r, l}^{(+)} g_{l, s}^{(-)}\right\} e^{-i E t}+\operatorname{Res}\left[G\left(r, s ; E_{l o c}\right)\right] e^{-i E_{l o c} t}
\end{gathered}
$$

where, within the integral, $E=2 h-J \cos \theta$, while

$$
g_{i, j}^{( \pm)}(E)=\frac{\alpha G_{0}^{( \pm)}(i, j ; E)}{1-\alpha G_{0}^{( \pm)}(l, l ; E)}=\frac{\alpha e^{ \pm i|\theta||i-j|}}{ \pm i \sin |\theta|-\alpha} .
$$

The amplitude $f_{r s}$ given in eq. (10) contains two terms. One contribution comes from the localized state; it has the same structure of the concurrence $C_{r s}$ given in eq. (92). The other contribution is written in terms of an integral over the branch cut region of the Green function, describing the evolution of the states within the continuous band. While the former goes to zero with the amplitude of the defect $\alpha$, the latter does not, as it reduces to the un-perturbed transition amplitude when $\alpha \rightarrow 0$. This is given by the Bessel function of order $r-s$ and argument $\tau=J t, J_{r-s}(\tau)$, see Ref. [16]. The main effect of a finite defect field in the second term is a distortion of the amplitude, with the appearance of contributions describing scattering at the defect during propagation from $s$ to $r$.

In the limit of $\alpha \gg 1$, a series of simple remarks can be drawn. First of all, the localization length goes to zero, and therefore the localized state is really concentrated on the impurity site. As a result, if the qubit residing there is initially entangled with an external one, the entanglement doesn't propagate at all, and the concurrence has the simple form (for $s=l=0$ ):

$$
C_{r} \simeq \begin{cases}1-\frac{1}{2 \alpha^{2}} & r=0 \\ \frac{r}{|\alpha| \tau} J_{r}(\tau) & r \neq 0\end{cases}
$$

Secondly, the transmission through the defect is very poor. Indeed, when the sender is on one side of the impurity (located at $l=0$ ), while the receiving site is on the other one, at a distance $d=|r|+|s|$, the concurrence is given by

$$
C_{r} \simeq \frac{1}{2|\alpha|}\left[J_{d+1}(\tau)+J_{d-1}(\tau)\right]
$$

which decays both with the distance between the sites and with time as $\tau^{-3 / 2}$.

Finally, the defect can reflect entanglement. Indeed, when both the sender and the receiver are on the same side (say $s, r>0$ with $r>s$ ), the concurrence is given by the combination of three terms:

$$
C_{r}=\left|(-1)^{s} J_{r-s}(\tau)-J_{r+s}-i \frac{J_{r+s+1}+J_{r+s-1}}{2 \alpha}\right| .
$$

This relation has a simple interpretation: the entanglement can either propagate directly from $s$ to $r$ (a process weighted by the un-perturbed transition amplitude given by the Bessel function $J_{r-s}$ ), or it can propagate to the defect and then be reflected to the site $r$ (thus travelling a distance $2 s+r-s=r+s$ ). This latter process has an amplitude obtained by $i$ ) taking the Bessel function $J_{r+s}$ which describes propagation along this distance, and 2) subtracting (with its own relative phase) the amplitude for entanglement transmission through the defect.

Although not easily seen from the full expression of eq. (10), these two main features of entanglement localization and reflection at the impurity site, are present also for moderate values of $\alpha$. This can be seen in Fig. (2), where the case $\alpha=-2$ is illustrated. By increasing $\alpha$, these effects become more and more pronounced and the secondary propagation lines which are present in Fig. (2) tend to disappear.

To better characterize the effect of the impurity on entanglement propagation, we can introduce reflection and transmission coefficients. Indeed, we can exploit the fact that states with one reversed spin saturate the CKW inequality 20], which in our case implies that 


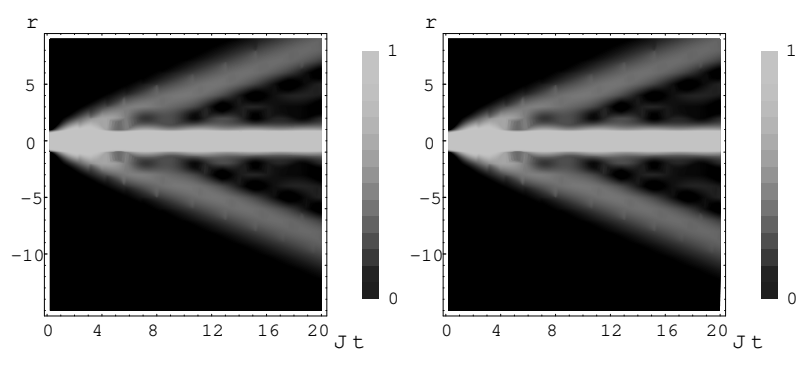

FIG. 2: Time evolution of the concurrence $C_{r}$ for $\alpha=-2$ and two different initial conditions. The left plot shows the case in which the sender resides at the defect site $(l=s=0)$, whereas the right plot shows the case $s=-5$. In the first case, the entanglement with the external qubit stays localized at the sender site, while in the second one, entanglement propagates almost symmetrically from the sender site up to the defect, where it is reflected back.

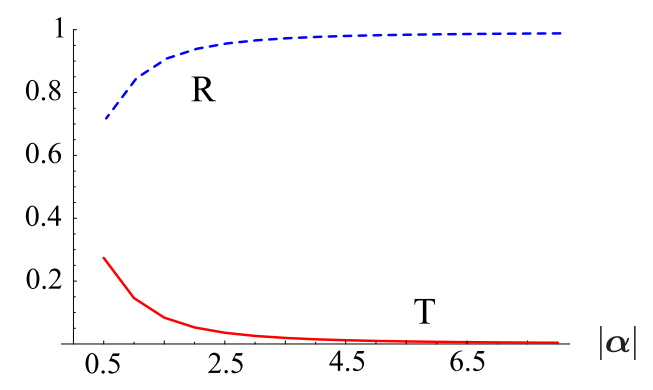

FIG. 3: Transmission and reflection coefficient as defined by Eq. (14), displayed as a function of $\alpha$.

$\sum_{r} C_{r}^{2}(t)=1$, and use the tangle as a probability distribution to define (to be specific, we consider the case of $s<0)$

$$
T=\lim _{t \rightarrow \infty} \sum_{r>0} C_{r}^{2}(t), \quad R=\lim _{t \rightarrow \infty} \sum_{r<0} C_{r}^{2}(t) .
$$

The transmission and reflection coefficients are shown in Fig. (3) as functions of $\alpha$.

Entanglement reflection at the defect is completely accounted for by the presence of the localized state. Indeed, since the overlap between the initial state and $\left|\Psi_{l o c}\right\rangle$ is exponentially small, entanglement propagates almost freely up to the defect site, but then the spin-reversal excitation cannot enter there. As the Hamiltonian describes nearest neighbor interaction, subsequent spins cannot receive the excitation and are, therefore, almost excluded from the dynamics. As a result, entanglement is reflected backwards.

In summary, we discussed the effect of a single magnetic field defect on entanglement in a spin chain. We found that this impurity produces a state with localized entanglement, whose presence strongly affects the dynamics of the chain. Indeed, the qubit at the defect site can either maintain entanglement for long time or work as an entanglement mirror. These two properties, obtained by modifying the local magnetic field of just one qubit, can be exploited to achieve a control on the propagation of pairwise quantum correlations in distributed qubit systems. Indeed, we have shown that entanglement can be stored for long times at the defect site and that its propagation can be modified so that it can be directed towards the receiver.

This work has been done within the framework of the PRIN2005029421 project.

[1] S. Bose, Phys. Rev. Lett. 91, 207901 (2003).

[2] A. Romito, R. Fazio, and C. Bruder, Phys. Rev. B 71, 100501 (2005); M. Paternostro, G. M. Palma, M. S. Kim, and G. Falci, Phys. Rev. A 71, 042311 (2005).

[3] F. Verstraete, M.A. Martìn-Delgado, and J. I. Cirac, Phys. Rev. Lett. 92087201 (2004).

[4] D. Burgarth, and S. Bose, Phys. Rev. A 71, 052315 (2005).

[5] V. Giovannetti and D. Burgarth, Phys. Rev. Lett. 96, 030501 (2006).

[6] M. Christandl, N. Datta, A. Ekert, and A. J. Landahl, Phys. Rev. Lett. 92, 187902 (2004); M. Christandl, N. Datta, T. C. Dorlas, A. Ekert, A. Kay, and A. J. Landahl, Phys. Rev. A 71, 032312 (2005); P. Karbach and J. Stolze, Phys. Rev. A 72, 030301(R) (2005).

[7] G. De Chiara, D. Rossini, S. Montangero, and R. Fazio, Phys. Rev. A 72, 012323 (2005).

[8] X. Wang, Phys. Rev. E 69, 066118 (2004).

[9] J. J. Fitzsimons, and J. Twamley, Phys. Rev. A 72, 050301 (2005).

[10] L.F. Santos, Phys. Rev. A 67, 062306 (2003); L.F. Santos, and G. Rigolin, Phys. Rev. A 71, 032321 (2005).

[11] O. Osenda, Z. Huang, and S. Kais, Phys. Rev. A 67, 062321 (2003).

[12] L.F. Santos, M.I. Dykman, M. Shapiro, and F.M. Izrailev, Phys. Rev. A 71, 012317 (2005).

[13] L.F. Santos, G. Rigolin, and C.O. Escobar, Phys. Rev A 69, 042304 (2004).

[14] P. W. Anderson, Phys. Rev. 109, 1492 (1958).

[15] E. N. Economou, "Green's Function in Quantum Physics" 2-nd edition, Springer-Verlag, Berlin (1983).

[16] L. Amico, A. Osterloh, F. Plastina, R. Fazio, and G. M. Palma, Phys. Rev. A, 69, 022304 (2004).

[17] V. Subrahmanyam, Phys. Rev. A, 69, 034304 (2004).

[18] L. F. Santos, and M. I. Dykman, Phys Rev. B 68, 214410 (2003).

[19] W. K. Wootters, Phys. Rev. Lett. 80, 2245 (1998).

[20] V. Coffman, J. Kundu, and W. K. Wootters, Phys. Rev. A 61, 052306 (2001). 


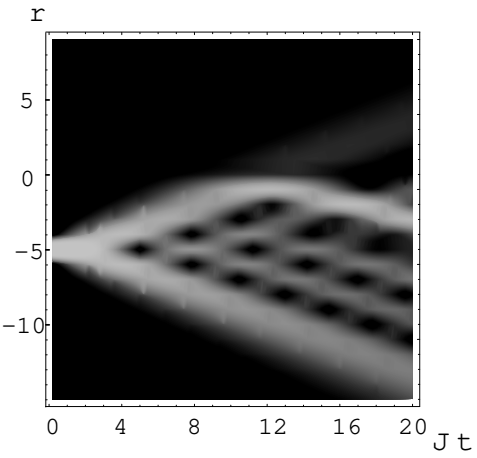

